\title{
Topology-preserving hexagonal thinning
}

\author{
Péter Kardos and Kálmán Palágyi* \\ Department of Image Processing and Computer Graphics, University of Szeged, \\ H-6720 Szeged, Árpád tér 2, Hungary
}

(Received 16 April 2012; revised version received 14 July 2012; accepted 17 August 2012)

\begin{abstract}
Thinning is a well-known technique for producing skeleton-like shape features from digital binary objects in a topology-preserving way. Most of the existing thinning algorithms work on input images that are sampled on orthogonal grids; however, it is also possible to perform thinning on hexagonal grids (or triangular lattices). In this paper, we point out to the main similarities and differences between the topological properties of these two types of sampling schemes. We give various characterizations of simple points and present some new sufficient conditions for topology-preserving reductions working on hexagonal grids.
\end{abstract}

Keywords: digital topology; topology preservation; thinning; hexagonal grids

2010 AMS Subject Classifications: 68U10; 68T10; 52C99; 68R99

\section{Introduction}

Various applications of image processing and pattern recognition are based on skeletons (i.e. region-based shape features) [19]. Thinning is an iterative layer-by-layer object reduction until only the skeletons of the binary objects are left [11]. Thinning algorithms in 2D are capable of extracting medial lines and topological kernels [6]. A topological kernel is a minimal set of points that is topologically equivalent to the original object $[6,9,10,17]$. Existing thinning algorithms are generally assuming orthogonal grids, but thinning algorithms working on hexagonal grids have also been proposed [3,4,7,20-22]. Besides the above results, a non-thinning-based hexagonal skeletonization approach is presented in [1], which is based on distance transform.

Parallel thinning algorithms are composed of reductions (i.e. some object points having a value of ' 1 ' in a binary picture that satisfy certain topological and geometric constrains are changed to '0' ones simultaneously) [5].

In the fields of digital topology and image processing, non-orthogonal grids have been studied by a number of authors $[2,10,15,18]$. A hexagonal grid, which is formed by a tessellation of regular hexagons, corresponds, by duality, to the triangular lattice, where the points are the centres of that hexagons (Figure 1). The advantage of hexagonal grids over the orthogonal ones lies in the fact that in hexagonal sampling scheme, each pixel is surrounded by six equidistant nearest

*Corresponding author. Email: palagyi@inf.u-szeged.hu

ISSN 0020-7160 print/ISSN 1029-0265 online (C) 2012 Taylor \& Francis

http://dx.doi.org/10.1080/00207160.2012.724198

http://www.tandfonline.com 


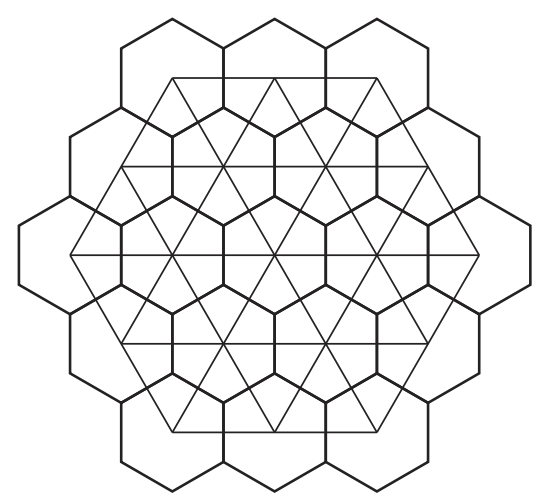

Figure 1. A hexagonal grid and the triangular lattice that is dual to it. Hexagonal pixels are bounded by thick line segments. Pixel centres (i.e. points in the triangular lattice) are joined with thin line segments.

neighbours, which results in a less ambiguous connectivity structure and in a better angular resolution compared with the rectangular case $[13,15]$. Wuthrich and Stucki [23] give also an algorithmic comparison between hexagonal and square-based models.

Topology preservation is an essential requirement for thinning algorithms [10]. In order to verify that a reduction preserves topology, Ronse [17], Kong [9], and Ma [14] gave some sufficient conditions for topology-preserving reductions working on the orthogonal grids. Later, Palágyi et al. proposed further sufficient conditions that consider individual pixels (instead of pixel configurations), and thus they are capable of designing deletion rules of topologically correct 3D thinning algorithms [16].

In our previous work [7] we showed a way to characterize simple pixels for the hexagonal case, and we proposed some sufficient conditions similar to Ronse's ones, which can be used to verify the topological correctness of hexagonal reductions. In this paper, we present further characterizations of simple pixels, and we give some new sufficient conditions that make possible to generate deletion conditions for various topology-preserving parallel thinning algorithms. In addition, two algorithms based on our new conditions are also proposed.

The rest of this paper is organized as follows. Section 2 reviews the basic notions of $2 \mathrm{D}$ digital hexagonal topology. Section 3 introduces various characterizations of simple pixels, which play a key role in the view of topology preservation. In Section 4 , we give our sufficient conditions for reductions to preserve topology. To illustrate the usefulness of these criteria, we propose in Section 5 two topology-preserving hexagonal parallel thinning algorithms, which are derived from our sufficient conditions. Finally, we round off the paper with some concluding remarks.

\section{Basic notions}

We use the fundamental concepts of digital topology as reviewed by Kong and Rosenfeld [10].

Let us consider the digital space $V$, and let us call the elements of $V$ pixels. We refer with the notation $N_{j}(p)$ the set of pixels that are j-adjacent to pixel $p$ and let $N_{j}^{*}(p)=N_{j}(p) \backslash\{p\}$. Note that reflexive and symmetric adjacency relations are generally considered (i.e. $p \in N_{j}(p)$ and if $q \in N_{j}(p)$, then $\left.p \in N_{j}(q)\right)$. The sequence of distinct pixels $\left\langle x_{0}, x_{1}, \ldots, x_{k}\right\rangle$ is called a $j$-path of length $k$ from pixel $x_{0}$ to pixel $x_{k}$ in a non-empty set of pixels $X \subseteq V$ if each pixel of the sequence is in $X$ and $x_{i}$ is $j$-adjacent to $x_{i-1}$ for each $1 \leq i \leq k$. Note that a single pixel is a $j$-path of length 0 . Two pixels are said to be $j$-connected in the set $X \subseteq V$ if there is a $j$-path in $X$ between them. A set of pixels $X \subseteq V$ is $j$-connected in the set of pixels $Y \supseteq X$ if any two pixels in $X$ are $j$-connected in $Y$. 


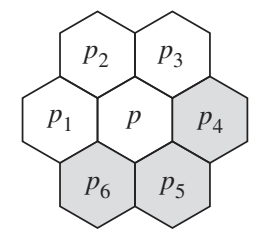

Figure 2. Indexing scheme for the elements of $N_{6}^{*}(p)$ on hexagonal grid. Pixels $p_{i}(i=4,5,6)$, for which $p \prec p_{i}$ holds, are shown in grey.
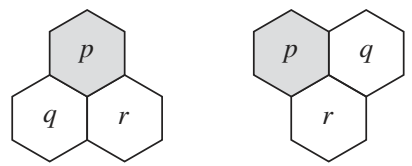

Figure 3. The two possible kinds of unit triangles. Since $p \prec q \prec r, p$ is the first element of the unit triangle.

An $(m, n)$ binary digital picture is a quadruple $\mathcal{P}=(V, m, n, B)$ [10]. Each pixel in $B \subseteq V$ is called a black pixel and has a value of 1 assigned to it. Each pixel in $V \backslash B$ is called a white pixel and has a value of 0 assigned to it. An object is a maximal $m$-connected set of black pixels, while a white component is a maximal $n$-connected set of white pixels.

A maximal set of mutually $m$-adjacent pixels is called a unit cell. Let us denote by $C(p)$ the number of objects in picture $\mathcal{P}=\left(V, m, n, B \cap N_{k}^{*}(p)\right)$, where $k=\max (m, n)$. A simple closed $m$-curve in an $(m, n)$ picture is a connected set of black pixels each of which is $m$-adjacent to exactly two other points in the set. A black pixel $p$ is called a border pixel in an $(m, n)$ picture if it is $n$-adjacent to at least one white pixel (i.e. $N_{n}(p) \backslash B \neq \emptyset$ ).

For 2D binary pictures sampled on the orthogonal lattice $\mathbb{Z}^{2}$, typically $(8,4)$ and $(4,8)$ pictures are considered. Unit cells on $(8,4)$ pictures (that contain four mutually 8 -adjacent pixels) are called unit squares.

In the hexagonal case $(6,6)$ pictures are generally considered, i.e. pictures given by the quadruple $(H, 6,6, B)$, where $H$ denotes the set of pixels in the hexagonal grid. Figure 2 shows the 6 -neighbours of a pixel $p$ denoted by $N_{6}(p)$. We use the precedence relation denoted by $\prec$ between two pixels $p$ and $q \in N_{6}(p)$ as follows:

$$
p \prec q \Leftrightarrow q \in\left\{p_{4}, p_{5}, p_{6}\right\} .
$$

(It is easy to see that this relation is irreflexive, antisymmetric, and transitive; therefore, it is a partial order on the set $N_{6}(p)$.) A unit cell in $(6,6)$ pictures is composed of three mutually 6adjacent pixels $p, q$, and $r$, which we call a unit triangle (Figure 3), where pixel $p$ is called the first element of that unit triangle.

An important topological property of digital pictures is formulated in the discrete Jordan's theorem, which states that if the black pixels of a picture form a simple closed curve, then there are exactly two white components in that picture $[10,15]$. This criterion does not hold for $(8,8)$ and $(4,4)$ pictures, but only for $(8,4)$ and $(4,8)$ pictures on orthogonal grids. (Note that in the $(4,8)$ case, the simple closed curve may not be contained in a unit square.) For resolving the connectivity paradoxes in the latter two cases, Latecki et al. have proposed a solution by introducing the notion of well-composedness [12]. A binary digital picture sampled on the orthogonal grid is well-composed, if every black or white 8-component is a 4-component, as well. Transforming digital pictures into well-composed pictures guarantees that the discrete Jordan's property will hold even for the $(8,8)$ and $(4,4)$ case. 
Interestingly, unlike $(4,4)$ and $(8,8)$ pictures, $(6,6)$ pictures satisfy the discrete Jordan theorem, due to the special structure of hexagonal grids [15]. As only one type of connectivity is considered, there is no need for that kind of correction of hexagonal binary pictures as described in [12], i.e. every $(6,6)$ picture is 'well-composed' in itself.

A reduction transforms a binary picture only by changing some black pixels to white ones (which is referred to as the deletion of 1's). A 2D reduction does not preserve topology if any object is split or is completely deleted, any white component is merged with another white component, or a new white component is created [9].

Besides the topological correctness, another key requirement of thinning is shape preservation. For this aim, thinning algorithms usually apply reductions that do not delete so-called end pixels that provide important geometrical information related to the shape of objects. We say that a black pixel $p$ is called an end pixel in a $(6,6)$ picture if it is 6 -adjacent to exactly one black pixel.

\section{Characterizations of simple pixels}

A simple pixel is a black pixel whose deletion is a topology-preserving reduction [10]. This property of a pixel can be verified in different ways. A useful characterization of simple pixels on $(8,4)$ and $(4,8)$ pictures is stated as follows:

THEOREM $3.1[10]$ Let $p$ be a non-isolated border point in a $\left(\mathbb{Z}^{2}, m, n, B\right)$ picture $((m, n) \in$ $\{(8,4),(4,8)\})$. Then the following are equivalent:

(1) $p$ is a simple point.

(2) $p$ is m-adjacent to just one $m$-component of $N_{8}^{*}(p) \cap B$.

(3) $p$ is n-adjacent to just one $n$-component of $N_{8}(p) \backslash B$.

For $(6,6)$ pictures, Kardos and Palágyi proved the following equivalency.

THEOREM 3.2 [7] Black pixel p in picture $(H, 6,6, B)$ is simple if and only if both of the following conditions are satisfied:

(1) $p$ is a border pixel.

(2) $C(p)=1$.

We show that the above results can be both written in the following general form.

THEOREM 3.3 Let $p$ be a non-isolated border point in a $(V, m, n, B)$ picture $((V, m, n) \in$ $\left.\left\{\left(\mathbb{Z}^{2}, 8,4\right),\left(\mathbb{Z}^{2}, 4,8\right),(H, 6,6)\right\}\right)$, and let $k=\max (m, n)$. Then the following are equivalent:

(1) $p$ is a simple point.

(2) $p$ is m-adjacent to just one m-component of $N_{k}^{*}(p) \cap B$.

(3) $p$ is n-adjacent to just one $n$-component of $N_{k}(p) \backslash B$.

Proof If $(V, m, n) \in\left\{\left(\mathbb{Z}^{2}, 8,4\right),\left(\mathbb{Z}^{2}, 4,8\right)\right\}$, then we get Theorem 3.1.

Let us suppose that $(V, m, n)=(H, 6,6)$ and let $p$ be a non-isolated border point. First, we prove that Conditions 1 and 2 are equivalent. By Theorem 3.2, pixel $p$ is simple if and only if $C(p)=1$. By the definition of the notation $C(p)$, this means that there is exactly one object in picture $\left(H, 6,6, B \cap N_{6}^{*}(p)\right)$. Note that, as $m=\max (m, n)=6$, this statement is equivalent with Condition 2. 
Now, we will see that Conditions 2 and 3 are equivalent. As $p$ is a non-isolated border point, there is at least 1 black and 1 white pixel in $N_{6}^{*}(p)$. As each pixel in $N_{6}^{*}(p)$ is 6-adjacent with exactly two other members of $N_{6}^{*}(p)$, it can be easily checked that the number of 'white to black' and 'black to white' transitions in the sequence of pixels $\left\langle p_{1}, p_{2}, p_{3}, p_{4}, p_{5}, p_{6}, p_{1}\right\rangle$ is equal. Furthermore, as each object of $N_{6}^{*}(p) \cap B$ and each white component of $N_{6}(p) \backslash B$ determines exactly one "white to black' and one 'black to white' transition in that sequence, we can also state that the number of objects in $N_{6}^{*}(p) \cap B$ equals with the number of white components in $N_{6}(p) \backslash B$, which implies the equivalency between Conditions 2 and 3 .

Note that in both of the orthogonal and hexagonal cases, the simplicity of pixel $p$ proves to be a local property, as it depends only on the pixels in $N_{8}(p)$ or $N_{6}(p)$ (depending on the type of picture).

Kong proposed an easily visualized characterization of simple pixels on conventional orthogonal images by using the concept of attachment sets [9]. Here, we give an adaptation of his model for the hexagonal case, using some notions from [9].

For this purpose, we consider a pixel $p$ to be a closed regular hexagon in the Euclidean plane (Figure 1). Let $U(p)=\left\{e_{1}, \ldots, e_{6}\right\}$ denote the boundary of that hexagon that is the union of its six edges, where pixels $p$ and $p_{i}$ share edge $e_{i}(i=1, \ldots, 6)$ (Figure 2). Two edges in the universe set $U(p)$ are adjacent if they share a vertex. A set of $n(n=0,1, \ldots, 6)$ edges $E \subseteq U(p)$ is connected, if its elements can be arranged in a sequence $\left\langle e_{i_{1}}, \ldots, e_{i_{n}}\right\rangle$ such that $e_{i_{k}}$ and $e_{i_{k+1}}$ are adjacent for each $k=1, \ldots, n-1$.

Let us suppose that $p$ is an object pixel in picture $\mathcal{P}=(H, 6,6, B)$. Then the $\mathcal{P}$-attachment set of $p$ is defined as follows:

$$
\mathcal{A}(p)=\left\{e_{i} \mid e_{i} \in U(p) \text { and } p_{i} \in B\right\} .
$$

Its complement $\overline{\mathcal{A}(p)}$ is defined as

$$
\overline{\mathcal{A}(p)}=U(p) \backslash \mathcal{A}(p) .
$$

Note that there exists a one-to-one correspondence between the possible $2^{6}$ kinds of configurations in $N_{6}^{\star}(p)$ and the possible attachment sets $\mathcal{A}(p)$. This property does not hold for $(8,4)$ pictures (i.e. the same attachment set may be assigned to numerous pixel configurations) [9]. Using the above terminology, we can state the following theorem, similarly as for the orthogonal case in [9]:

TheOREM 3.4 Let $\mathcal{P}=(H, 6,6, B), p \in B$, and let $\mathcal{A}(p)$ be the $\mathcal{P}$-attachment set of $p$. Then, $p$ is simple in $\mathcal{P}$ if and only if both $\mathcal{A}(p)$ and its complement, $\overline{\mathcal{A}(p)}$, are non-empty and connected.

Proof Let us suppose that $p$ is simple. Then, from Theorem 3.2 it follows that $C(p)=1$. This implies that in the hexagonal grid model of $\mathcal{P}$, the union of the hexagons representing the pixels of $N_{6}^{*}(p) \cap B$ forms a connected set in the Euclidean plane, which also means that the edges of these hexagons contained also in $p$ form a connected set, as well, and obviously this connected set coincides with $\mathcal{A}(p)$. As $C(p) \neq 0, p$ is not an isolated object pixel, therefore, $\mathcal{A}(p)$ is non-empty. It is not hard to see that from $C(p)=1$ follows that the pixels of $N_{6}^{*}(p) \backslash B$ form a single object in the picture $\left(H, 6,6, N_{6}^{*}(p) \backslash B\right)$, hence $\overline{\mathcal{A}(p)}$ is also connected. By Theorem 3.2, $p$ is a border pixel, i.e. $N_{6}^{*}(p) \backslash B$ is non-empty, therefore, $\overline{\mathcal{A}(p)}$ must be also non-empty.

As Theorem 3.2 gives an equivalent property of simplicity, the proof for the case when $p$ is not simple can be proved by using a very similar train of thoughts as above.

We note that it is not hard to see that if $\mathcal{A}(p)$ in Theorem 3.4 is connected, then so does $\overline{\mathcal{A}(p)}$, hence it is sufficient to check this connectivity for $\mathcal{A}(p)$ in order to decide the simplicity of $p$.

Figure 4 shows some illustrative examples for Theorem 3.4. 
(a)

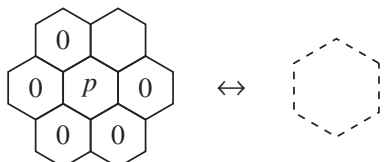

(c)

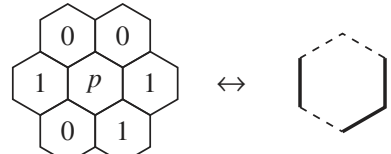

(b)

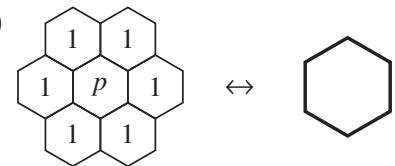

(d)

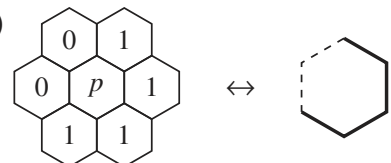

Figure 4. Examples for the $\mathcal{P}$-attachment sets. $p$ is simple only in (d), as in the other cases $\mathcal{A}(p)=\emptyset$ (a), or $\overline{\mathcal{A}(p)}=\emptyset$ (b), or both $\mathcal{A}(p)$ and $\overline{\mathcal{A}(p)}$ are not connected (c). The thick line segments represent elements of $\mathcal{A}(p)$, while the points covered by dashed line segments belong to $\overline{\mathcal{A}(p)}$.

\section{Sufficient conditions for topology-preserving reductions}

Reductions delete a set of black pixels and not only a single simple pixel. Kardos and Palágyi gave the following sufficient conditions for topology-preserving reductions on hexagonal grids.

THEOREM 4.1 [7] A reduction $\mathcal{O}$ is topology preserving in picture $(H, 6,6, B)$, if all of the following conditions hold:

(1) Only simple pixels are deleted by $\mathcal{O}$.

(2) If $\mathcal{O}$ deletes two 6-adjacent pixels $p, q$, then $p$ is simple in $(H, 6,6, B \backslash\{q\})$, or $q$ is simple in $(H, 6,6, B \backslash\{p\})$.

(3) $\mathcal{O}$ does not delete completely any black component contained in a unit triangle.

We note that this is an adaptation of Ronse's sufficient conditions for $(8,4)$ and $(4,8)$ pictures $[14$, 17], which can be formulated as follows.

THEOREM $4.2[14]$ A reduction $\mathcal{O}$ is topology preserving in picture $\left(\mathbb{Z}^{2}, m, n, B\right)((m, n) \in$ $\{(8,4),(4,8)\})$, if all of the following conditions hold:

(1) Only simple pixels are deleted by $\mathcal{O}$.

(2) For any two n-adjacent black pixels, $p, q \in B$ that are deleted by $\mathcal{O}, p$ is simple in $\left(\mathbb{Z}^{2}, m, n, B \backslash\{q\}\right)$, or $q$ is simple in $\left(\mathbb{Z}^{2}, m, n, B \backslash\{p\}\right)$.

(3) if $(m, n)=(8,4), \mathcal{O}$ never deletes any object contained in a unit square.

Furthermore, we state that Condition 3 of Theorem 4.1 can be simplified so that we get the following result equivalent to Theorem 4.1 .

THEOREM 4.3 A reduction $\mathcal{O}$ is topology preserving in picture $(H, 6,6, B)$, if all of the following conditions hold:

(1) Only simple pixels are deleted by $\mathcal{O}$.

(2) If $\mathcal{O}$ deletes two 6-adjacent pixels $p, q$, then $p$ is simple in $(H, 6,6, B \backslash\{q\})$, or $q$ is simple in $(H, 6,6, B \backslash\{p\})$.

(3) $\mathcal{O}$ does not delete completely any object that forms a unit triangle.

Proof Let us examine objects containing less then 3 black pixels. $\mathcal{O}$ does not delete an isolated black pixel by Condition 1, as it is not a simple pixel. Let us consider an object composed by 
exactly two black pixels, $p$ and $q . p$ is an isolated pixel in $(H, 6,6, B \backslash\{q\})$ and vice versa; therefore, $\mathcal{O}$ does not delete any object containing exactly two pixels by Condition 2 .

Consequently, the verification of the above kinds of objects is unnecessary in Condition 3 of Theorem 4.1, hence $\mathcal{O}$ fulfils Conditions $1-3$ of our theorem if and only if it satisfies Conditions 1-3 of Theorem 4.1 .

While the above results state conditions for pixel-configurations, we can derive from Theorem 4.3 some new criteria that examine if an individual pixel is deletable or not. Here, we give two alternatives of such conditions, and then we compare their characteristics.

THEOREM 4.4 A reduction $\mathcal{O}$ is topology preserving in picture $(H, 6,6, B)$, if each pixel p deleted by $\mathcal{O}$ satisfies the following conditions:

(1) $p$ is a simple pixel in $(H, 6,6, B)$.

(2) For any simple pixel $q \in N_{6}^{*}(p)$, p is simple in $(H, 6,6, B \backslash\{q\})$, or q is simple in $(H, 6,6, B \backslash\{p\})$.

(3) $p$ is not an element of an object that forms a unit triangle.

Proof Let us suppose that $S$ is the set of pixels deleted by $\mathcal{O}$. By Condition $1, S$ contains only simple pixels; therefore, Condition 1 of Theorem 4.3 is also satisfied. Let $p, q \in S$ be two arbitrary 6-adjacent pixels. Then, according to Condition $2, p$ is simple in $(H, 6,6, B \backslash\{q\})$ or $q$ is simple in $(H, 6,6, B \backslash\{p\})$. This means that Condition 2 of Theorem 4.3 holds, as well. We show that Condition 3 of Theorem 2 also holds. $\mathcal{O}$ does not delete a single pixel object by Condition 1 . Objects composed by two 6-adjacent black pixels may not be completely deleted by Condition 2. Finally, from Condition 3 it follows that each element of any object composed by 3 mutually 6-adjacent pixels is retained by $\mathcal{O}$.

THEOREM 4.5 A reduction $\mathcal{O}$ is topology preserving in picture $(H, 6,6, B)$, if each pixel p deleted by $\mathcal{O}$ satisfies the following conditions:

(1) $p$ is a simple pixel in $(H, 6,6, B)$.

(2) For any simple pixel $q \in N_{6}^{*}(p)$ for which $p \prec q$, p is simple in $(H, 6,6, B \backslash\{q\})$, or $q$ is simple in $(H, 6,6, B \backslash\{p\})$.

(3) $p$ is not the first element of an object that forms a unit triangle.

Proof Again, let $S$ be the set of pixels deleted by $\mathcal{O}$. Similarly as it was seen for Theorem 4.3, Condition 1 of Theorem 4.3 is satisfied. Let $p, q \in S$ be again two arbitrary 6-adjacent pixels. If $p$ is simple in $(H, 6,6, B \backslash\{q\})$ or $q$ is simple in $(H, 6,6, B \backslash\{p\})$, then we get Condition 2 of Theorem 4.3, which corresponds to Condition 2 of Theorem 4.3. Let us suppose that for two 6-adjacent pixels $p, q \in S p$ is not simple in $(H, 6,6, B \backslash\{q\})$ and $q$ is not simple in $(H, 6,6, B \backslash\{p\})$. In this case, by Condition $2, q \prec p$ holds. But this would mean that $q$ does not satisfy Condition 2 , hence $q \notin S$, which leads to a contradiction. Therefore, this situation cannot occur.

We can show the same way as for the previous theorem that $\mathcal{O}$ does not delete objects composed by less than three black pixels. Furthermore, by Condition 3, exactly one element of any object composed of 3 mutually 6 -adjacent pixels is retained.

We remark that, by examining only three-pixel objects in Condition 3 , Theorem 4.5 can be considered as a stronger version of Theorem 3 in [8].

To illustrate the difference between Theorems 4.3 and 4.5 , we define two reductions.

Definition 4.6 Let $\mathcal{R}_{1}$ be the reduction that deletes all pixels satisfying all conditions of Theorem 4.3 from any $(6,6)$ picture. 
(a)

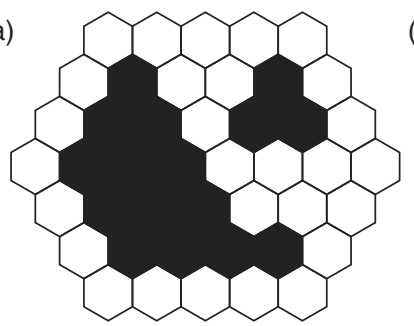

(b)

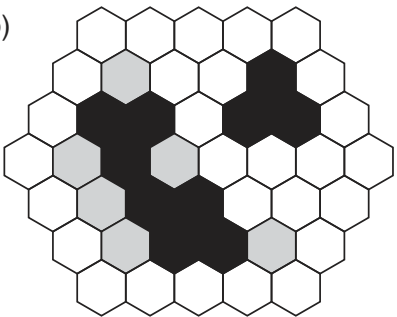

(c)

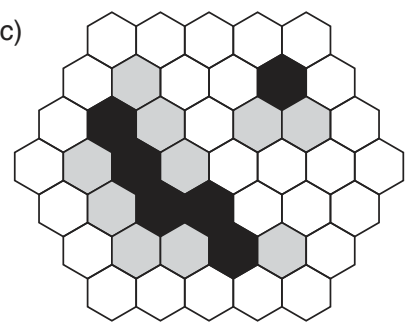

Figure 5. The original picture (a) and the results produced by the reductions $\mathcal{R}_{1}$ (b) and $\mathcal{R}_{2}$ (c) on it. Deleted pixels are depicted in grey.

(a)

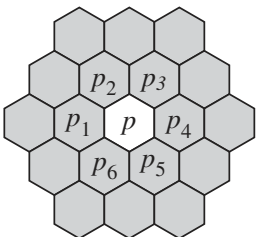

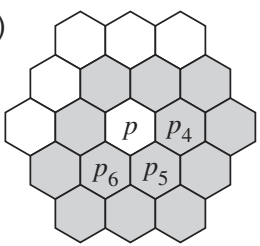

Figure 6. The supports of the reductions $\mathcal{R}_{1}$ (a) and $\mathcal{R}_{2}$ (b). Pixels in the supports are depicted in grey.

DEFINITION 4.7 Let $\mathcal{R}_{2}$ be the reduction that deletes all pixels satisfying all conditions of Theorem 4.5 from any $(6,6)$ picture.

Let us perform these reductions on a small hexagonal binary image containing two objects shown in Figure 5(a). The effects of operators $\mathcal{R}_{1}$ and $\mathcal{R}_{2}$ can be observed in Figures 5(b)-(c). The example in Figure 5 reveals that reductions derived from the conditions of Theorem 4.5 can delete more simple pixels than the ones satisfying the conditions of Theorem 4.3. This could be a drawback if we wanted to construct thinning algorithms from operators like $\mathcal{R}_{1}$, as skeleton-like shape features in the ideal case should not contain any simple pixel. From this view, $\mathcal{R}_{2}$ may be preferred to $\mathcal{R}_{1}$. However, it is also easy to see that the criteria in Theorem 4.5 take into account the direction of $p$ relative to the simple pixels of $N_{6}^{*}(p)$, while this is not the case for Theorem 4.3. From this follows that, unlike $\mathcal{R}_{2}, \mathcal{R}_{1}$ is invariant on rotations by $60^{\circ}$ and $120^{\circ}$, and this geometric property can be considered as another important aspect of thinning. Thus, it is not self-evident which conditions should be applied for composing thinning algorithms, as it strongly depends on the field of application.

Let us examine the supports (i.e. the minimal sets of pixels whose values determine the new values of pixels [5]) of reductions $\mathcal{R}_{1}$ and $\mathcal{R}_{2}$. By Theorem 3.3, the simplicity of a black pixel $p$ required by Condition 1 of Theorem 4.3 is determined by the elements of $N_{6}^{*}(p)$. Furthermore, for the verification of Condition 2 of Theorem 4.3, we also need to find out if the black pixels in $N_{6}(p)$ are simple or not, which means that we need to check the elements of $\bigcup_{i=1}^{6} N_{6}\left(p_{i}\right)$ (note that $N_{6}(p)$ is included in this set). Similarly, Condition 3 of Theorem 4.3 can be verified by exactly the same elements as above. The difference between Theorems 4.3 and 4.5 is that, by the definition of the relation $\prec$, Conditions 2 and 3 of the latter theorem only take into account the pixels in $\bigcup_{i=4}^{6} N_{6}\left(p_{i}\right)$ and $N_{6}(p)$. Consequently, the supports of reductions $\mathcal{R}_{1}$ and $\mathcal{R}_{2}$ contain the pixels depicted in grey in Figure 6.

\section{Thinning algorithms derived from sufficient conditions for topology preservation}

In this part, we give two thinning algorithms HT-1 and HT-2 that are derived from the previously introduced reductions $\mathcal{R}_{1}$ and $\mathcal{R}_{2}$, respectively (see Algorithm 1). These algorithms are based 
on the fully parallel thinning scheme presented in [6], in which the same reduction operation is applied in each phase of the thinning process.

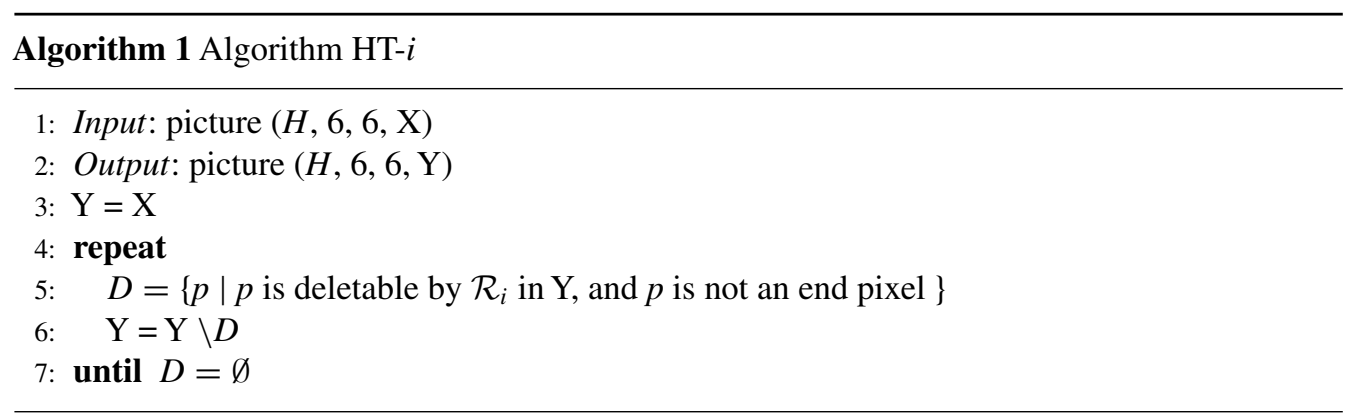

The kernel of the repeat cycle in Algorithm 1 corresponds to one iteration step. All pixels in the set of deletable pixels $D$ are deleted simultaneously. HT- $i$ terminates when stability is reached $(i=1,2)$. In an iteration step of the proposed algorithms, only black pixels that are not end pixels may be deleted. As we mentioned in Section 2, end pixels play a key role in shape preservation, which makes possible to produce the medial lines of objects.

Note that, as $\mathcal{R}_{1}$ and $\mathcal{R}_{2}$ fulfil the conditions of Theorems 4.3 and 4.5 , it is self-evident that they preserve topology.

(a)

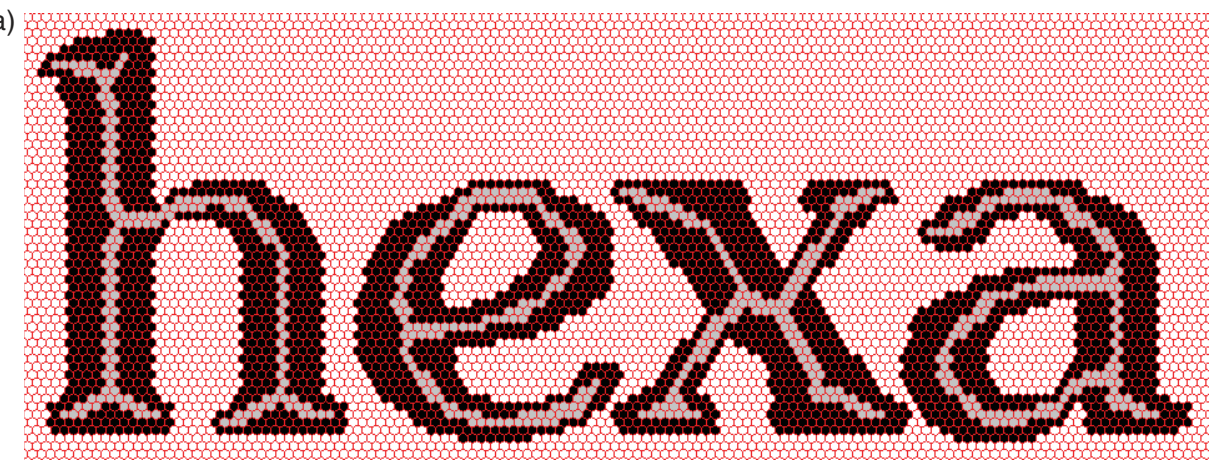

HT-1

(b)

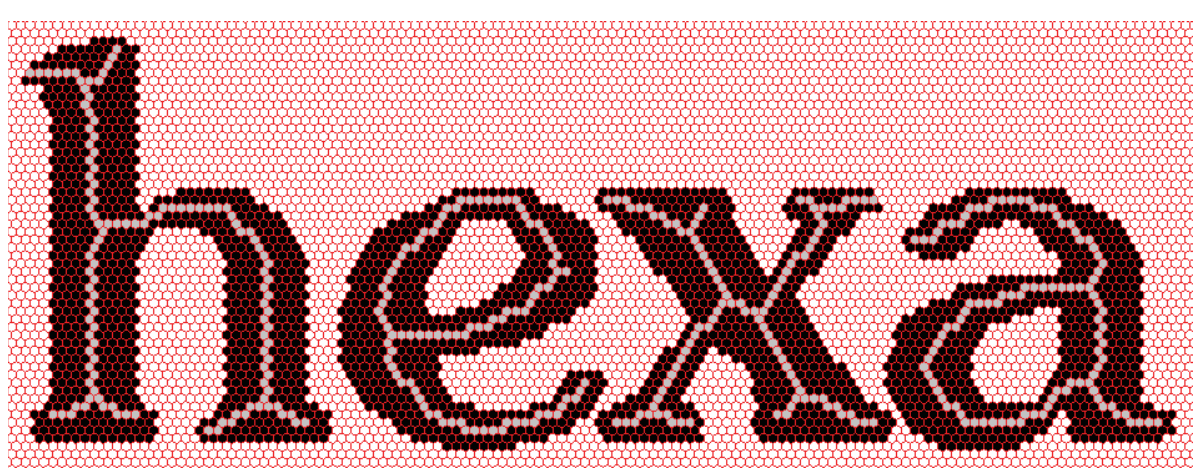

HT-2

Figure 7. Thinning of some characters sampled on hexagonal grids. Medial lines produced by Algorithm 1 are superimposed on the original objects. 
In experiments, our fully parallel algorithms were tested on objects of various pictures. Here, we present an illustrative example below (Figure 7). By comparing the results of HT-1 and HT-2 in Figure 7, we can observe that algorithm HT-2 deletes more object pixels than algorithm HT-1. As the deleting conditions of $\mathcal{R}_{1}$ are stricter than the ones of $\mathcal{R}_{2}$, algorithm HT-1 cannot alter some 2-pixel wide segments.

We remark that by using our characterization of end pixels and by combining our sufficient conditions for topology preservation with various thinning strategies we can derive further thinning algorithms, similar to the results in $[8,16]$.

\section{Conclusions}

In this work we have compared some of the relationships of 2D digital topology between pictures sampled on orthogonal and hexagonal grids. For $(6,6)$ pictures, we have also introduced some characterizations of simple pixels and some new sufficient conditions for topology-preserving reductions. The main novelty of the proposed conditions lies in the fact that they are based on the verification of individual pixels, unlike the existing criteria which examine pixel configurations. It might be a quite challenging task to prove topology preservation for hexagonal algorithms like the ones presented in [4,21], as their deletion conditions are determined by several matching templates or Boolean formulae whose verification usually requires a number of cases to deal with. Our conditions, however, are capable of constructing hexagonal thinning algorithms whose topological correctness is automatically (i.e. without the need of any proof) guaranteed.

\section{Acknowledgements}

This research was supported by the European Union and the European Regional Development Fund under the grant agreements TÁMOP-4.2.1/B-09/1/KONV-2010-0005 and TÁMOP-4.2.2/B-10/1-201-0012, and the grant CNK80370 of the National Office for Research and Technology (NKTH) and the Hungarian Scientific Research Fund (OTKA).

\section{References}

[1] G. Borgefors and G. Sanniti di Baja, Skeletonizing the Distance Transform on the Hexagonal Grid, Proceedings of 9th International Conference on Pattern Recognition, Rome, Italy, 1988, pp. 504-507.

[2] V.E. Brimkov and R.P. Barneva, Analytical honeycomb geometry for raster and volume graphics, Comp. J. 48 (2005), pp. 180-199.

[3] E.S. Deutsch, On parallel operations on hexagonal arrays, IEEE Trans. Comp. C-19 (1970), pp. $982-983$.

[4] E.S. Deutsch, Thinning algorithms on rectangular, hexagonal, and triangular arrays, Commun. ACM 15 (1972), pp. 827-837.

[5] R. Hall, Parallel connectivity-preserving thinning algorithms, in Topological Algorithms for Digital Image Processing, T.Y. Kong and A. Rosenfeld, eds., Elsevier Science Inc., New York, NY, 1996, pp. 145-179.

[6] R.W. Hall, T.Y. Kong, and A. Rosenfeld, Shrinking binary images, in Topological Algorithms for Digital Image Processing, T.Y. Kong and A. Rosenfeld, eds., Elsevier Science Inc., New York, NY, 1996, pp. 31-98.

[7] P. Kardos and K. Palágyi, On Topology Preservation for Hexagonal Parallel Thinning Algorithms, Proceedings of the International Workshop on Combinatorial Image Analysis, Lecture Notes in Computer Science, Vol. 6636, Springer, Berlin, 2011, pp. 31-42.

[8] P. Kardos and K. Palágyi, Hexagonal Parallel Thinning Algorithms Based on Sufficient Conditions for Topology Preservation, Proceedings of the Computational Modeling of Objects Presented in Images: Fundamentals, Methods and Applications III, Rome, Italy, 2012, pp. 63-68.

[9] T.Y. Kong, On topology preservation in 2-d and 3-d thinning, IJPRAI 9 (1995), pp. 813-844.

[10] T.Y. Kong and A. Rosenfeld, Digital topology: Introduction and survey, Comput. Vision Graph. Image Process. 48 (1989), pp. 357-393. Available at http://dl.acm.org/citation.cfm?id=71397.71400.

[11] L. Lam, S. Lee, and C. Suen, Thinning methodologies - a comprehensive survey, IEEE Trans. Pattern Anal. Mach. Intell. 14 (1992), pp. 869-885.

[12] L. Latecki, U. Eckhardt, and A. Rosenfeld, Well-composed sets, Comput. Vis. Image Understanding 61 (1995), pp. 70-83.

[13] M. Lee and S. Jayanthi, Hexagonal Image Processing: A Practical Approach (Advances in Pattern Recognition), Springer-Verlag, New York, Inc., Secaucus, NJ, 2005. 
[14] C. Ma, On topology preservation in 3d thinning, CVGIP 59 (1994), pp. 328-339.

[15] S. Marchand-Maillet and Y.M. Sharaiha, Binary Digital Image Processing - A Discrete Approach, Academic Press, New York, 2000

[16] K. Palágyi, G. Németh, and P. Kardos, Topology preserving parallel 3D thinning algorithms, in Digital Geometry Algorithms. Theoretical Foundations and Applications to Computational Imaging, R.P. Barneva and V.E. Brimkov, eds., Springer, Berlin, 2012, pp. 165-188.

[17] C. Ronse, Minimal test patterns for connectivity preservation in parallel thinning algorithms for binary digital images, Discrete Appl. Math. 21 (1988), pp. 67-79. Available at http://www.sciencedirect.com/science/article/pii/ $0166218 X 8890$.

[18] J. Serra, Image Analysis and Mathematical Morphology, Vol. 1, Academic Press, London, 1982.

[19] K. Siddiqi and S. Pizer, Medial Representations: Mathematics, Algorithms and Applications, 1st ed., Springer Publishing Company, Incorporated, Berlin, 2008.

[20] R. Staunton, An analysis of hexagonal thinning algorithms and skeletal shape representation, PR 29 (1996), pp. 1131-1146.

[21] R. Staunton, A one pass parallel hexagonal thinning algorithm, 1999. Seventh International Conference on Image Processing and Its Applications (Conf. Publ. No. 465), Vol. 2, Manchester, UK, 1999, pp. 841-845.

[22] P. Wiederhold and S. Morales, Thinning on quadratic, triangular, and hexagonal cell complexes., IWCIA'08, Buffalo, NY, 2008, pp. 13-25.

[23] C. Wuthrich and P. Stucki, An algorithm comparison between square- and hexagonal-based grids, Graphical Models Image Process. 53 (1991), pp. 324-339. 